\title{
Biological motion and animacy belief induce similar effects on involuntary shifts of attention
}

\author{
Ned Chandler-Mather ${ }^{1} \cdot$ Timothy Welsh $^{2} \cdot$ Samuel Sparks $^{1} \cdot$ Ada Kritikos $^{1,2}$ \\ Published online: 14 August 2019 \\ (C) The Psychonomic Society, Inc. 2019
}

\begin{abstract}
Biological motion is salient to the human visual and motor systems and may be intrinsic to the perception of animacy. Evidence for the salience of visual stimuli moving with trajectories consistent with biological motion comes from studies showing that such stimuli can trigger shifts of attention in the direction of that motion. The present study was conducted to determine whether or not top-down beliefs about animacy can modify the salience of a nonbiologically moving stimulus to the visuomotor system. A nonpredictive cuing task was used in which a white dot moved from a central location toward a left- or right-sided target placeholder. The target randomly appeared at either location 200,600, or 1,300 ms after the motion onset. Five groups of participants experienced different stimulus conditions: (1) biological motion, (2) inverted biological motion, (3) nonbiological motion, (4) animacy belief (paired with nonbiological motion), and (5) computer-generated belief (paired with nonbiological motion). Analysis of response times revealed that the motion in the biological motion and animacy belief groups, but not in the inverted and nonbiological motion groups, affected processing of the target information. These findings indicate that biological motion is salient to the visual system and that top-down beliefs regarding the animacy of the stimulus can tune the visual and motor systems to increase the salience of nonbiological motion.
\end{abstract}

Keywords Biological motion $\cdot$ Animacy $\cdot$ Attention $\cdot$ Social IOR $\cdot$ Action observation

The ability to interpret, understand, and predict the action of another person is thought to be fundamental to our functioning as social beings (Blakemore \& Decety, 2001). Central to this ability are processes that enable us to distinguish between kinematic patterns that represent human and nonhuman motion. In early work on these processes, Johansson (1973) placed lights on the joints and distal locations of limbs of a human model and recorded the displacement of these lights in the dark while the model performed actions such as walking and running. When watching such point-light displays (PLDs), observers can discriminate animate (biological) from inanimate motion, and can distinguish between different human models (Alaerts, Nackaerts, Meyns, Swinnen, \& Wenderoth, 2011; Johansson, 1973). It has been suggested that observers use the kinematic information (e.g., position

Ada Kritikos

a.kritikos@psy.uq.edu

1 School of Psychology, University of Queensland, St Lucia, Queensland, Australia

2 Centre for Motor Control, Faculty of Kinesiology \& Physical Education, University of Toronto, Toronto, Ontario, Canada displacement, direction, velocities, and acceleration) of the dots in relation to each other as critical low-level information to perceive action and intuit animacy in the motion of the set of dots representing the model (e.g., Johansson, 1973; Kilner, Hamilton, \& Blakemore, 2007). Furthermore, on the basis of differences in the relative trajectories of the moving dots, human observers can intuit the emotional state (Alaerts et al., 2011; Atkinson, Dittrich, Gemmell, \& Young, 2004; Clarke, Bradshaw, Field, Hampson, \& Rose, 2005), gender (Troje, 2002), and identity (Cutting \& Kozlowski, 1977) of the model. Thus, the perception of biological motion may be critical to a wide range of social cognitive processes.

One important component of living and working in social environments is the ability to understand the actions of other individuals and predict their intentions and goals. This ability allows observers to coordinate their own actions with those of other people. Even static images of body parts, such as pointing hands (Ulloa, Marchetti, Taffou, \& George, 2015) or eye gaze (Bayliss et al., 2013; Ulloa et al., 2015) toward targets, are salient cues that are associated with reorientation of attention. When people view animate stimuli, previous research has revealed both overt (Flanagan \& Johansson, 2003) and covert (Bardi, Di Giorgio, Lunghi, Troje, \& Simion, 2015; 
Shi, Weng, He, \& Jiang, 2010; Wang, Yang, Shi, \& Jiang, 2014) shifts of attention to the location of potential targets when biological motion patterns are directed toward that location (see also Welsh et al., 2005; Welsh, Higgins, Ray, \& Weeks, 2007). The shift of the observer's attention toward the target of the model's action may indicate that animate motion is salient, and the observer uses this cue to anticipate and predict how the model may interact with and use the target object to achieve a specific goal (Atkinson, Simpson, \& Cole, 2018; Flanagan \& Johansson, 2003).

In one article reporting a shift in attention to a potential forthcoming target location, Shi et al. (2010) centrally presented a PLD of a human appearing to walk in a left- or rightward direction. The figure appeared to walk toward the left or right of the screen, but did not actually translate (i.e., it remained centrally located). After a brief exposure to the walking figure, participants experienced a left- or right-tilted Gabor patch that appeared randomly on the left or right side of the screen. Although the figure did not translate spatially in either direction and did not predict the location and orientation of the Gabor patch, participants had shorter response times when the Gabor patch appeared in the walking direction of the figure than when the Gabor patch appeared in the direction opposite to the walking direction. This facilitation for processing objects in the walking direction was absent when the same human PLD was inverted, when the figure was static, and when the figure involved rotary motion (Shi et al., 2010; see also Bosbach, Prinz, \& Kerzel, 2004). Overall, a series of studies have indicated that biological motion is salient to the human visual systems and that biological motion in the environment can lead to a shift of attention to locations in the direction of the biological motion in advance of the model actually reaching that location. This shift of attention based on biological motion may be conceptualized as an attempt to understand and predict the interaction that the model will have with any potential object located in the direction of that motion (e.g., Flanagan \& Johansson, 2003).

Interestingly, and relevant to the stimulus used in this investigation, the shift of attention during observation of movement can occur with minimal motion information. For example, in follow-up work to Shi et al. (2010), Wang et al. (2014) reported that exposure to just two white dots representing the movement of a point-light walker's feet is sufficient to trigger an attention shift and response facilitation in the walking direction. Importantly, this facilitation emerges both when participants were naïve to the animacy of the dots (not given any specific instructions about them) and when they are told explicitly that the dots represent human walking. Crucially, the magnitude of the effect is larger and more robust when participants believe the dots represent human motion than when they are naïve (Wang et al., 2014). This difference in the magnitude of the effect raises an important question regarding the distinct contributions of higher- and lower-level factors.
Lower-level kinematic features of stimuli, such as motion perception, may drive shifts of attention, but higher-level beliefs in the animacy of the motion may shape or prime the systems involved in perceiving biological motion, action, and guidance of attention. The relative contributions of these factors are unclear, because both have typically been either present or absent in previous paradigms examining attentional shifts. For example, Flanagan and Johansson (2003) asked participants to observe the grasping actions of a human or robotic arm - thus implying a clear distinction between human and nonhuman kinematic patterns as well as beliefs in animacy between the human and robot conditions. Similarly, Wang et al. had participants observe the motion of two dots derived from the feet of a walking human. Participants either were naïve or were explicitly informed about the human derivation of the stimuli- that is, the same kinematic patterns of actual walking were used, with the potential for varied beliefs about the animacy of the moving dots.

A belief in stimulus animacy appears to enhance responses to low-level (Wang et al., 2014) and complex (Sparks, Sidari, Lyons, \& Kritikos, 2016; Stanley, Gowen, \& Miall, 2007) displays, as well as enhancing motor performance (i.e., motor contagion; Gowen, Bolton, \& Poliakoff, 2016; Longo \& Bertenthal, 2009; Sparks, Sidari, et al., 2016; Stanley et al., 2007). However, the role that beliefs about animacy may play in tuning or biasing the visual and motor systems to increase the salience of nonbiological motion and to generate shifts of attention is less clear. In this article, we focus on how animacy (biological motion) and the belief that a nonbiological stimulus is actually animate could alter the salience of the moving stimulus and, as a result, performance in an attention task. Thus, a shift of attention, indexed by changes in response times (RTs) to targets presented in a cue-target task, was exploited in the present study as a means of determining whether a belief of animacy can tune the visual system to increase the visuomotor salience of a nonbiological motion stimulus.

\section{The present research}

To recap, the direction of biological motion induces shifts in attention to locations in advance of the motion (Shi et al., 2010; Wang et al., 2014). It is possible that belief may likewise tune the visual system to increase the salience of nonbiological motion (Stanley et al., 2007). As a result of this beliefinduced increase in salience, a nonbiological motion signal may generate shifts of attention in a manner similar to those shifts of attention that precede actual biological motion of a stimulus to a target. The present experiment was designed to test this prediction by examining the patterns of RTs that emerge for targets presented after noninformative motion cues. Participants saw a dot moving from a central location 
toward one of two potential target locations, on the left or the right side of the visual display. The direction of motion of the dot did not predict the location of the subsequent target. Because this series of investigations is part of ongoing work on modulations in attention in action-centered (Song \& Nakayama, 2009; Tipper, Lortie, \& Baylis, 1992; Welsh \& Weeks, 2010; see also Hommel et al., in press) and jointaction (e.g., social inhibition of return; Welsh et al., 2005; Welsh, Lyons, et al., 2007; Welsh, Chandler-Mather, Sparks, \& Kritikos, 2019) contexts, participants made reaching movements from a starting point on a keyboard to a target on a monitor positioned in front of them. We instructed participants to respond as soon as possible after the target (a green square) had appeared.

For separate groups of participants, the motion of the single dot was a biological motion pattern (the movement of a human index finger reaching toward the left or right target location) or was nonbiological, transient motion. Among the groups that viewed nonbiological motion, one group was told that this motion represented a human movement, whereas the other groups were not given any instructions regarding the nature of the motion (similar to Wang et al., 2014, and Stanley et al., 2007). By assessing the patterns of RTs that emerged in the groups that observed a nonhuman trajectory under different animacy belief instructions, we were able to determine whether top-down belief in the animacy of a stimulus was sufficient to the prime of the visual system to generate a shift of attention.

A key feature of the present design was that the target was presented randomly 200,600 , or $1,300 \mathrm{~ms}$ after the onset of the cue. These stimulus onset asynchronies (SOAs) were used to provide insights into the time course and potential mechanisms associated with any attentional shifts associated with the onset of the motion cue toward a target. There is a wellestablished pattern of RTs to targets presented randomly at cued and uncued locations as a function of the SOA (Posner \& Cohen, 1984; see Klein, 2000, for a review; see also Neyedli \& Welsh, 2012). Typically, even though the cue does not predict the target location, RTs to targets presented at a saliently cued location are shorter than RTs to targets at the uncued location when the SOA is short $(<200 \mathrm{~ms})$. This facilitatory effect at the cued location likely occurred because the salient cue led to a shift of attention to the cued location, facilitating the processing of the target information at that location relative to the uncued location. When the SOA is extended (> $300 \mathrm{~ms}$ ), however, RTs to cued targets are longer than RTs to uncued targets. It is thought that these longer RTs at the cued location occur because the participant knows that the cue was not predictive of the target locations and so, with increasing SOA, disengages attention from the cued target location and shifts attention back to the central fixation point before the appearance of the target. Crucially, this disengagement is said to involve the establishment of an inhibitory code at the cued location. This inhibitory code briefly hinders the return of attention, and thus the processing of the target at the cued location. As a result, RTs for targets at the cued location are longer than those for targets at the previously uncued location, the inhibition of return (IOR) effect (Posner \& Cohen, 1984; Tipper, Weaver, Jerreat, \& Burak, 1994). In the present study, the facilitation/IOR paradigm was used to index whether or not any of the given stimuli and/or instructions tuned the visual system such that attention was shifted to a potential target location by the moving dot representing human and nonhuman motion.

Participants in the biological group observed a digitized dot motion pattern derived from the video recording of a female model reaching and pointing to an object placed at the left or the right of the screen. Consistent with canonical pointing movements, the trajectory of the single dot curved upward vertically and moved with a velocity that steadily increased in the early stages of the movement, reached a peak near the middle of the movement, and then decreased toward the end of the movement. We predicted that the RTs for the group watching this biologically derived motion would be consistent with the pattern of facilitation/inhibition following a shift of attention to the cue (e.g., Posner \& Cohen, 1984)that is, shorter RTs for cued than for uncued targets at brief SOAs, but longer RTs for cued than for uncued targets at longer SOAs. This pattern of RTs is expected to emerge on the basis of previous evidence that the human visual system is tuned to biological (human) motion toward a location and that this motion can shift attention to that target location (e.g., Flanagan \& Johansson, 2003; Shi et al., 2010; Wang et al., 2014). Indeed, a series of studies have revealed IOR after a person observes the reaching movements of another person to a lateral target location (e.g., Welsh et al., 2005; Welsh, Lyons, et al., 2007; Welsh, McDougall, \& Weeks, 2009; but see Atkinson, Simpson, Skarratt, \& Cole, 2014; Doneva, Atkinson, Skarratt, \& Cole, 2017). Thus, if the motion of the dot in the biological condition is perceived as biological motion and derived from a human model, the pattern of facilitation and inhibition associated with a shift of attention to the cued location should be observed.

Participants in the inverted-biological group observed a dot with the same velocity profile, but whose canonical trajectory was inverted about the horizontal axis such that it moved initially in a downward direction before moving upward toward the target (see Chang \& Troje, 2008; Shi et al., 2010; Wang et al., 2014). Participants in the nonbiological group observed a dot that moved in a nearly straight line (did not curve vertically) with a constant velocity profile. Finally, the dot for the participants in the animacy belief group moved with the same characteristics as the dot in the nonbiological condition (i.e., in a straight line with a constant velocity profile), but participants were told that the motion of the dot actually represented the real movement of a human model. 
If only human motion in a canonical trajectory orientation and with a human velocity profile is salient and can generate a shift of attention, regardless of belief in animacy, then the pattern of facilitation and inhibition should only emerge for biological group. Conversely, if any motion (biological or nonbiological) is salient and sufficient to shift of attention toward a forthcoming target, then the pattern of RT facilitation for the brief SOA (200 $\mathrm{ms}$ ), and inhibition for the longer SOAs (600 and 1,300 ms) should emerge in all groups. If shifts of attention depend on a biological motion profile, regardless of the veridical orientation of the trajectory curve, then the pattern of RT facilitation and inhibition should emerge for the biological and invertedbiological groups, but not for the nonbiological and animacy belief groups. Finally, as regards belief in the animacy of the motion, if belief can tune or prime the visual system to increase the visuomotor salience of the moving stimulus to generate a shift of attention, then the pattern of RT facilitation and inhibition should emerge for the animacy belief group but not for the nonbiological group.

\section{Method}

\section{Participants}

A total of 84 first-year psychology students (age range 17 to 34 years, $M=19.54$ years, $S D=3.72$ ) from the University of Queensland participated for course credit. All participants were female and had normal or corrected-to-normal vision. Only female participants were tested, because there is evidence that females are more tuned to social cues (Sparks, Sidari, et al., 2016), such as gaze (Bayliss, di Pellegrino, \& Tipper, 2005). A male experimenter ran the sessions. Participants provided verbal consent and were compensated with course credit. Each participant was allocated alternately into one of the conditions, in the order biological, nonbiological, inverted, animacy belief. The procedures were approved by the University of Queensland School of Psychology Ethics Committee.

\section{Apparatus and stimuli}

Participants sat at a table $(60 \times 60 \mathrm{~cm})$ in front of a monitor (Samsung UA55D6600; 55-in., resolution 1,920 × 1,080 pixels, $55 \mathrm{~Hz}$ ). The monitor was aligned such that it was parallel with the participant's coronal plane. A keyboard (polling rate $=1000$ $\mathrm{Hz}$ ) was positioned in front of the participant, aligned with the coronal plane, and fixed to the table using Velcro straps. Black cloth covered the keyboard, such that the keyboard itself was not visible to the participant. A piece of circular white foam (diameter $=3 \mathrm{~cm}$ ) was glued on the cloth positioned over the "0" key and functioned as the "home button." Participants pressed on the white foam to hold this button down with their right index finger. The tip of each participant's index finger was approximately $30 \mathrm{~cm}$ from the screen surface.

The total area of the visual display on the monitor was $680 \mathrm{~mm}$ in height $\times 536 \mathrm{~mm}$ wide. This consisted of a black area $(680 \times 216 \mathrm{~mm})$ on which the stimuli appeared, bordered on the right and left by nontransparent gray bars $(680 \times 160$ $\mathrm{mm}$ ) that occluded a portion of the motion of the dot as it neared the potential target locations (see Fig. 1). Images of

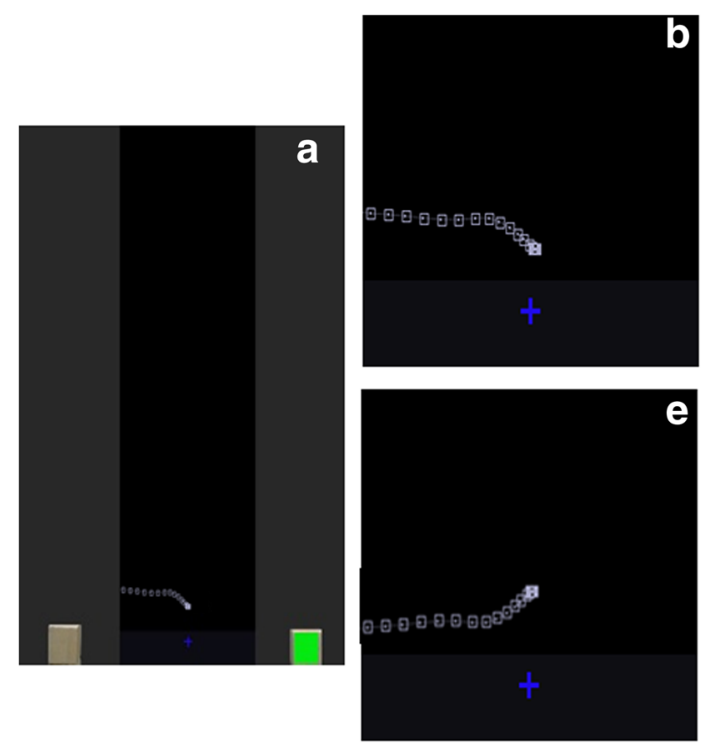

Fig. 1 (A) Display of the blue fixation cross, a white dot stimulus at its origin, the subsequent biological motion trajectory, and the target placeholders. The gray occluders on the left and right of the display obscured the final section of the dot's trajectory. The motion profile of
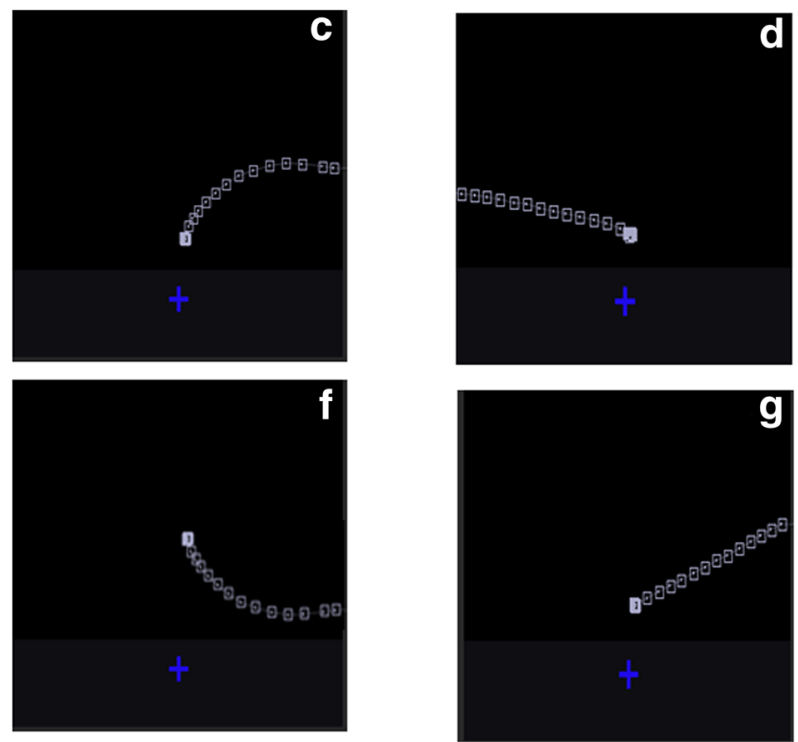

the dot cue moved with biological motion to the left (B) or the right (C), inverted biological motion to the left (D) or the right (E), and nonbiological motion to the left $(\mathrm{F})$ or the right $(\mathrm{G})$. Each white square indicates the location of the dot in a single frame 
balsawood cubes $(50 \times 55 \mathrm{~mm})$ appearing to sit on the frame of the monitor at the center of the bottom edge of each occluder. These images were the placeholders for the targets. At the start of each trial, the white dot (diameter $=14 \mathrm{~mm}$ ) appeared concurrently on the black background positioned $10 \mathrm{~mm}$ above a centrally positioned blue fixation cross $(8 \times$ $8 \mathrm{~mm}$ ) that was aligned with the participant's mid-sagittal plane. The distance between the center of the blue cross and the edge of the occluder was $106 \mathrm{~mm}$. The targets were green squares $(37 \times 37 \mathrm{~mm})$ that appeared for $100 \mathrm{~ms}$ on top of one of the target locations in each trial (see Fig. 1A).

To create the biological-motion stimuli (Figs. 1B and 1C), we recorded a female model performing two pointing actions with her right index finger, beginning at her mid-sagittal plane and terminating at wooden blocks located at lateral locations on her left and right (the approximate locations of the subsequent targets in the main experimental task). For each left- and rightward action, Adobe After Effects CC 2014 was used to track the displacement of the index fingernail of the model in action. Specifically, the location of the nail of the index finger of the moving right hand was digitized on each frame with a white dot and this digitized white dot was used as the stimulus.

To create the nonbiological motion stimuli for the right and left reaches (Figs. 1F and 1G), the displacement of the dot on each frame was altered to form a linear trajectory and velocity profile, such that the dot was displaced with equal distances and transition angles from frame to frame. The inverted motion trajectory (Figs. 1D and 1E) was created by transposing the minimum and maximum $y$-coordinates of the upright motion trajectory. The initial $y$-coordinate of each inverted motion trajectory, moving toward the left or right of the display, was the same as the final $y$-coordinate of the upright, biological motion trajectory. The $x$-coordinates were the same in both conditions. Thus, apart from the starting and finishing positions of the inverted motion, this dot followed the same trajectory and velocity profile as the biological motion. However, whereas the upright biological motion trajectory curved slightly upward as it progressed away from the central staring position and toward the lateral target, the inverted trajectory curved slightly downward. The duration of all dot motion in the left direction was 200 $\mathrm{ms}$, and the duration of dot motion to the right was $167 \mathrm{~ms}$. This small discrepancy in movement times emerged because the dot motion was based on the trajectory of a human model's right index finger starting from a mid-sagittal location. Thus, the model's rightward movement (and participant's right) was within her right hand's hemispace and shorter, but her leftward movement (and participant's left) meant that the right hand crossed to the left hemispace and was thus lengthened.

\section{Procedure}

Participants were tested individually. Upon arrival, participants were seated before the monitor and given verbal and written instructions, which were different for each group (see below), that they would see a white dot moving across the screen, and then a green square would briefly flash in either the left or the right bottom part of the screen. At the start of each trial, participants were instructed to hold down a "home" button using the index finger of their dominant hand and to maintain fixation on the central cross. Participants were instructed that they could only break fixation after they had detected the target. They were instructed that their task was to respond as quickly and accurately as possible to the green flash (target) by releasing their finger from the start button and touching the location of the target on the monitor. The white dot was positioned above the fixation cross at the beginning of each trial. After a variable interval $(1,400,1,500$, or $1,600 \mathrm{~ms}$ ), the white dot's displacement was initiated toward either the left or the right side of the screen.

The direction of the dot motion cued the target location on $50 \%$ of trials. Importantly, participants were told that the dot direction did not predict target location. After a pseudorandomly varying interval (SOA: 200,600 , or $1,300 \mathrm{~ms}$ ) from dot movement initiation onset, the target appeared for $100 \mathrm{~ms}$. Participants responded by lifting their index finger from the home button and touching the target location on the screen. The RT was defined as the time (in milliseconds) taken for participants to release the home button after the target was presented on the screen. Participants pointed to the target and then returned their finger to the home button, pressing it down, and a new trial began after 1,500 ms.

The participants in the biological, nonbiological, and inverted-biological groups were not given any specific instructions regarding the nature of the motion stimuli they observed. The participants in the animacy belief group were informed verbally that they would see a white dot moving on the screen and that the motion of the white dot was based on a prerecorded pointing action of a female $\mathrm{PhD}$ student at the university. Participants were shown a still image that depicted a female model with her right index finger at a starting position and then two still images of her pointing to a wooden block on her right and then two stills of her pointing to a wooden block on her left. They were told that the experimenters had masked this image with a black screen and had marked her index finger with a white dot, so when the white dot moved it was following the pointing action of the model. In reality, the dot moved with nonbiological motion in the animacy instruction condition. Importantly, these still images were not present during the trials in the experiment.

After the instructions, all participants were given 12 practice trials, and then they completed two blocks of 60 experimental trials. Participants were given a brief break after the practice trials and between blocks. Cue type (biological, nonbiological, inverted biological, and animacy belief) was varied between participants, while target location (cued and uncued) and SOA (200, 600, 
and 1,300 ms) were randomized within each of the two experimental blocks within participants.

\section{Results}

RTs that were under 100 or over $1,000 \mathrm{~ms}$ were classified as anticipation and inattention errors, respectively, and were excluded from the analyses (126 of a possible 9,840 trials, $1.28 \%$ of total trials). Two participants (one each from inverted-biological and animacy belief groups) were excluded from analyses due to a failure to follow instructions - one participant recorded over $50 \%$ of the total responses as errors, and one participant persistently performed reaching responses to the cued location, not the target location. For the remaining 82 participants, an average of 118.46 (98.7\%) of a possible 120 trials were analyzed.

Response termination errors were not analyzed, because none of the 82 participants included in the study made any accuracy errors - all movements terminated on the correct target location. Possibly there were no response termination errors because any error in the initial direction of the pointing response in relation to the target location could be corrected during the course of the action (e.g., Welsh \& Elliott, 2004; Welsh, Elliott, \& Weeks, 1999). As a result of these online corrections, the response always ended on the correct target location.

Data from the biological $(N=21)$, inverted-biological $(N=$ $20)$, nonbiological $(N=21)$, and animacy belief $(N=20)$ groups were submitted to a mixed analysis of variance (ANOVA) with group as the between-subjects factor and target location (cued, uncued), target side (left, right), and SOA $(200,600,1,300 \mathrm{~ms})$ as within-subjects factors. We included target side because participants were responding only with their right hand and arm, and moving into ipsilateral and contralateral space, thus executing biomechanically different movements. Furthermore, previous work involving aiming movements in selective-attention paradigms has revealed that distracting stimuli in the space ipsilateral to the moving limb can generate greater interference effects than distractors in contralateral space (e.g., Meegan \& Tipper, 1998; Tipper et al., 1992). Such side-of-space effects are thought to emerge because movements into ipsilateral space are more efficient to plan and execute than movements to contralateral space. Hence, nontarget movements into ipsilateral space may cause greater interference than those into contralateral space because those ipsilateral movements require more time and resources to withhold and inhibit during the selection process than do contralateral movements (see Welsh \& Weeks, 2010, for review). Thus, target side was included as a factor in the original design to determine whether such side-of-space effects would emerge in the present cue-target task.
The main effect of group was not statistically significant, $F(3,78)=0.70, p=.555, \eta_{\mathrm{p}}{ }^{2}=.026$. A significant main effect of target location, $F(1,78)=6.52, p=.013, \eta_{\mathrm{p}}^{2}=.077$, revealed that RTs were longer for cued $(M=421.55, S D=$ 36.40) than for uncued $(M=418.96, S D=36.58)$ targets. There was a significant main effect of target side, $F(1,78)=$ $13.32, p<.001, \eta_{\mathrm{p}}{ }^{2}=.146$, showing that RTs to targets were longer for left-sided $(M=423.71, S D=36.54)$ than for rightsided $(M=416.79, S D=37.86)$ targets. We also found a significant main effect of SOA, $F(2,156)=200.06, p<$ $.001, \eta_{\mathrm{p}}{ }^{2}=.719$. Pairwise comparisons showed that RTs to targets were significantly longer for SOAs of $200 \mathrm{~ms}$ than for either 600-ms SOAs, $t(81)=19.52, p<.001(M=442.43, S D$ = 39.96; $M=407.76, S D=36.08$, respectively), or $1,300-\mathrm{ms}$ SOAs, $t(81)=14.48, p<.001(M=410.57, S D=36.57)$. The difference in RTs between trials with a $600-\mathrm{ms}$ and a $1,300-\mathrm{ms}$ SOA was not statistically significant, $t(81)=1.58, p=.117$.

Finally, we observed a significant three-way Group $\times$ Target Location $\times$ SOA interaction, $F(6,156)=2.94, p=$ $.010, \eta_{\mathrm{p}}{ }^{2}=.102$. To understand this interaction, a series of separate analyses were conducted on the data from each group. Because target side did not interact with any other factor, the data were collapsed across target sides for this analysis. These mean RTs were then submitted to a two-factor repeated measures ANOVA with target location and SOA as the factors, and to a series of $t$ tests.

The ANOVA of the RTs from the biological group (see Fig. 2A) revealed a statistically significant Target Location $\times$ SOA interaction, $F(2,40)=20.09, p<.001, \eta_{\mathrm{p}}^{2}=.501$. Post-hoc analysis of this interaction involved a series of planned comparisons using Holm-Bonferroni-corrected paired-sample $t$ tests, in which the RTs on cued and uncued target trials were compared at each SOA. Consistent with our hypotheses, RTs were significantly shorter on cued than on uncued trials at the 200-ms SOA $(M=436.90, S D=38.83 ; M=449.19, S D=$ 43.00 , respectively), $t(20)=3.290, p<.004$. By contrast, RTs were significantly longer on cued than on uncued target trials with SOAs of $600 \mathrm{~ms}, t(20)=4.7193, p<.000(M=412.68$, $S D=37.85 ; M=399.66, S D=36.83$, respectively), and 1,300 $\mathrm{ms}, t(20)=3.6163 .84, p=.002(M=415.62, S D=36.73 ; M=$ 406.66, $S D=40.35$, respectively) (see Fig. 2A). This pattern of differences is consistent with the patterns of faciltation and inhibition associated with attentional shifts generated by salient cues (e.g., Posner \& Cohen, 1984) and with social IOR following the observation of another person's aiming movement (e.g., Welsh et al., 2005).

The analysis of the RTs in the inverted and nonbiological conditions (see Figs. 2B and 2C, respectively) suggest that these motion stimuli did not generate shifts of attention. Specifically, the Target Location $\times$ SOA interaction was not statistically significant for inverted-biological group, $F(2,38)$ $=2.412, p=.103, \eta_{\mathrm{p}}{ }^{2}=.113$. Furthermore, although the ANOVA of the RTs from the nonbiological group revealed a 

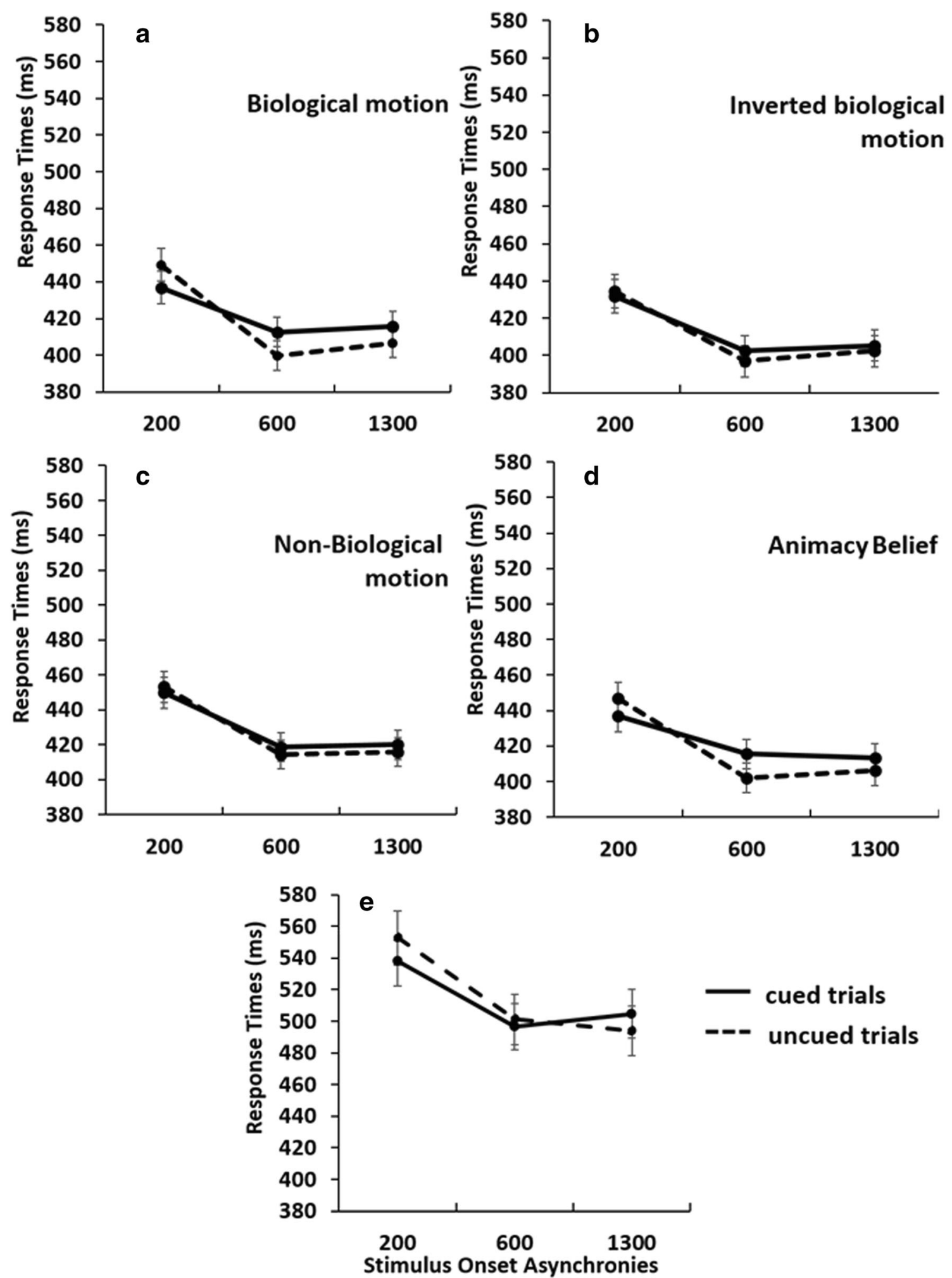

Fig. 2 Mean response times (with bars for standard errors of the means) for participants in the (A) biological motion, (B) inverted-biological motion, (C) nonbiological motion, (D) animacy belief, and (E) computer- generated belief conditions for SOAs of 200, 600, and 1,300 ms. Filled lines indicate cued trials, and dashed lines indicate uncued trials 
statistically significant Target Location $\times$ SOA interaction, $F(2,40)=3.380, p=.044, \eta_{\mathrm{p}}{ }^{2}=.145$, post-hoc analysis of this interaction using the same Holm-Bonferroni-corrected paired-sample $t$ tests did not reveal any significant differences between RTs to cued and uncued targets at any of the SOA conditions. That is, there was no statistically significant difference between the RTs on cued and uncued trials at SOAs of $200 \mathrm{~ms}, t(20)=1.420, p=.171(M=449.76, S D=44.49 ; M=$ 453.12, $S D=41.96$, respectively); $600 \mathrm{~ms}, t(20)=1.826, p=$ $.083(M=418.67, S D=40.00 ; M=414.30, S D=38.28$, respectively); or 1,300 ms, $t(20)=1.759, p=.094(M=$ 419.84, $S D=37.71 ; M=415.82, S D=39.42$, respectively). Thus, the interaction emerged because the positive cueduncued RT differences at the longer SOAs were statistically different from the negative cued-uncued RT differences at the short SOA, and not because any specific cuing effect was statistically significant. These results are consistent with the prediction that the dots with the nonbiological motion patterns were not sufficiently salient to generate shifts of attention.

Finally, the analysis of the animacy belief group (see Fig. 2D) revealed a significant Target Location $\times \mathrm{SOA}$ interaction, $F(2,38)=12.05, p<.001, \eta_{\mathrm{p}}{ }^{2}=.388$. As in the biological motion group, post-hoc analysis of the animacy belief group revealed that RTs were smaller on cued than on uncued trials with a 200-ms SOA, $t(19)=2.996, p=.007(M=437.00, S D$ $=44.38 ; M=446.97, S D=43.19$, respectively). In contrast, RTs were longer on cued than on uncued trials with SOAs of $600 \mathrm{~ms}, t(19)=2.559, p=.019(M=415.46, S D=39.35 ; M=$ 402.11, $S D=39.84$, respectively), and $1,300 \mathrm{~ms}, t(19)=3.310$ ,$p=.004(M=413.11, S D=39.64 ; M=405.97, S D=34.61$, respectively). These analyses indicate that, unlike in the nonbiological condition that used the same dot motion display, a shift of attention to a potential target location was generated by the moving dot for the animacy belief group.

Although the initial omnibus ANOVA revealed statistically significant differences in the patterns of RTs in the different conditions (as indicated by the significant three-way Group $\times$ Target Location $\times$ SOA interaction), a subsequent analysis was conducted in order to compare the nonbiological motion and animacy belief conditions directly (Figs. 2C and 2D, respectively). These two groups were specifically compared because they only differed in the instructions that created a belief that the dot motion is biological. Specifically, we looked for a significant three-way interaction between target location (cued, uncued), SOA (200, 600, 1,300 ms), and group (nonbiological motion and animacy belief). For the comparison between these two conditions, the main effect of group was not statistically significant, $F(1,39)=0.51, p=.481, \eta_{\mathrm{p}}{ }^{2}=$ .013 . There was a nonsignificant (though trending) main effect of target location, $F(1,1)=3.45, p=.071, \eta_{\mathrm{p}}{ }^{2}=.071$. We found a significant main effect of target side, $F(1,39)=6.73, p$ $=.013, \eta_{\mathrm{p}}{ }^{2}=.147$, such that RTs to targets were longer for leftsided $(M=427.96, S D=26.93)$ than for right-sided $(M=$
420.72, $S D=27.89)$ targets. We also found a significant main effect of SOA, $F(2,39)=87.52, p<.0001, \eta_{\mathrm{p}}{ }^{2}=.692$. Pairwise comparisons showed that RTs to targets were significantly longer for SOAs of $200 \mathrm{~ms}$ than for either $600-\mathrm{ms}$ SOAs, $t(40)=8.49, p<.0001(M=441.405, S D=42.65 ; M$ $=415.53, S D=39.76$, respectively), or 1,300-ms SOAs, $t(40)$ $=7.08, p<.0001(M=415.03, S D=38.64)$. The difference in RTs between trials with a $600-\mathrm{ms}$ and a $1,300-\mathrm{ms}$ SOA was not statistically significant, $t(40)=0.705, p=.882$. The interaction of interest, Target Location $\times \mathrm{SOA} \times$ Group, was also significant, $F(2,78)=3.556, p=.035, \eta_{\mathrm{p}}{ }^{2}=.084$. We take this result as more evidence that, in this task, animacy belief increased the visuomotor salience of the moving stimulus and moderated performance for cued versus uncued trials at the SOA intervals we implemented.

\section{Additional control condition}

It is possible that, in the animacy belief condition, the pattern of results could be attributable to the fact that participants were given verbal instructions regarding the cue stimulus, rather than to the meaning of the instructions specifically; that is, participants' awareness of the dot might have been heightened simply by the process of receiving instructions. ${ }^{1}$ Thus, we implemented a final control condition. In this computergenerated belief condition, participants were presented with a nonbiological motion cue and were told, prior to commencing the task, that the dot they were about to see had been generated by a computer. If a heightened awareness of the dot through instructions ("The dot was computer-generated, by the way ...") increased the salience of the stimulus and primed the visuomotor system, then the cue in this condition should lead to a pattern of RTs identical to that in the animacy belief condition - a facilitation effect at the 200-ms SOA and inhibition of return at the longer SOAs (600 and 1,300 ms).

Twenty-seven participants (age range 17 to 28 years, $M=$ 20.185 years, $S D=2.095$ ) from the University of Queensland completed the task. As in the main conditions, all participants were female and had normal or corrected-to-normal vision. A male experimenter ran the sessions. Participants provided verbal consent and were compensated with course credit. The procedures were approved by the University of Queensland School of Psychology Ethics Committee. The apparatus, stimuli, and procedure were identical to those aspects of the animacy belief condition, but the verbal instruction this time was that the dot had been computer-generated.

Two participants were excluded from the analysis for not following instructions, leaving a total of 25 participants included. Fifty trials $(1.39 \%)$ were excluded as anticipation errors or inattention errors.

\footnotetext{
${ }^{1}$ We thank an anonymous reviewer for this suggestion.
} 
Analysis of the RTs from the computer-generated belief group revealed no statistically significant main effect of target location, $F(1,24)=1.038, p=.319, \eta_{\mathrm{p}}{ }^{2}=.071$, but that there was a statistically significant main effect of SOA, $F(2,48)=$ $100.278, p<.0001, \eta_{\mathrm{p}}{ }^{2}=.807$. Post-hoc comparisons indicated that RTs were significantly shorter for the $600-\mathrm{ms}$ SOA condition than for the 200-ms SOA, $t(24)=14.28, p<.0001$ $(M=496.76, S D=73.14 ; M=537.82, S D=77.83$, respectively). In contrast, the RTs at the 600-ms SOA were not significantly different from those at the 1,300-ms SOA, $t(24)=$ $0.111, p=.972(M=496.76, S D=73.14 ; M=504.79, S D=$ 78.03, respectively).

Of greater relevance, we found a significant Target Location $\times$ SOA interaction, $F(1,24)=9.75, p<.0001, \eta_{\mathrm{p}}{ }^{2}$ $=.289$ (see Fig. 2E). Post-hoc analysis revealed that RTs were significantly shorter on cued than on uncued trials at the 200ms SOA, $t(24)=2.94, p=.007(M=537.82, S D=77.83 ; M=$ $552.79, S D=85.76$, respectively). In contrast, at the $600-\mathrm{ms}$ SOA, there was no significant difference in RTs, $t(24)=0.879$, $p=.388(M=496.76, S D=73.14 ; M=501.13, S D=80.61$, respectively). Finally, RTs were significantly longer on cued than on uncued trials at the 1,300-ms SOA, $t(24)=4.187, p<$ $.0001(M=504.79, S D=78.03 ; M=493.84, S D=78.86$, respectively).

Overall, the pattern of RTs in this computer-generated belief condition appear to be similar, but not identical, to those in the animacy belief condition; specifically, IOR was present at both the 600-ms and 1,300-ms SOAs in the animacy belief conditions, whereas IOR was only present at the $1,300-\mathrm{ms}$ SOA in the computer-generated condition. To test whether the patterns of RTs in these two conditions were different, a subsequent $2 \times 2 \times 3$ mixed ANOVA was conducted in which condition (animacy, computer-generated belief) was a between-subjects factor and target location and SOA were repeated measures factors. This ANOVA revealed a significant three-way interaction between condition, target location, and SOA, $F(2,86)=3.718, p=.028, \eta_{\mathrm{p}}{ }^{2}=.080$, confirming that the patterns of RTs in the two conditions were indeed different. The key difference between the conditions was that the cued-uncued RT difference was larger in the 600-ms SOA condition in the animacy belief condition than in the computer-generated belief condition, $t(43)=2.44, p=.019$. The magnitudes of the cued-uncued RT differences were not statistically different at the 200-ms SOA, $t(43)=0.78, p=.44$, and the 1,300 -ms SOA, $t(43)=1.09, p=.028$.

In conclusion, the results from this additional condition suggest that providing instructions about the nature of the dot motion seems to increase the visuomotor salience of the moving dot and may alter the manner in which the moving dot and other stimuli are processed. However, the series of comparisons between the RTs in the two instruction conditions revealed that when participants are given instructions regarding the nature of the motion, the moving dot believed to be human is nonetheless prioritized and processed differently from the one believed to be computer generated.

\section{Discussion}

In the present study, we varied the SOA $(200,600$, and 1,300 ms) between a nonpredictive cue and target to investigate whether a cue moving with biological rather than nonbiological motion triggers a shift in attention to a target located on the left or the right of the monitor. In particular, we wished to investigate whether belief that the transient nonbiological motion of a cue stimulus was generated by a human model (animacy belief) alters the priority and processing of the cue and causes the same shifts in attention as a biological stimulus. The results indicate two overarching findings. First, for the biological motion condition, we obtained the typical pattern of shorter RTs to the target at cued locations at brief SOAs, but longer RTs for cued targets (i.e., an IOR effect) for the extended SOAs. Therefore, we postulate that the task and the movement of the dot toward the cued target location engaged attentional and visuomotor processes, including engagement, disengagement, and inhibition of attention, as well as targetdirected movements through space. Second, and more importantly, the salience, processing, and subsequent modulation of attention were similar in conditions of biological motion (i.e., actual animacy) and animacy belief (nonbiological motion with an animacy instruction). The pattern of RTs that indexed increased salience of the moving dot was not evident in the nonbiological motion condition without belief, or when we disrupted the biological motion profile by inversion. Most importantly, although the results of the computer-generated belief condition suggest that the mere presence of instructions may have influenced the processing of the moving stimulus, instructions leading to belief in animacy of the moving dot still led to a different pattern of results. That is, IOR was absent for the 600-ms SOA, but was evident for the 1,300-ms SOA. Therefore, we suggest that in the context of slowed responses, there is still evidence that IOR can be abolished at the shorter 600-ms SOA. Thus, we postulate that we can disambiguate two mechanisms through which motion exerts an influence on attention - through bottom-up visuomotor processes, operationalized here as biological motion, and through top-down/ higher-level conceptualizations, such as beliefs regarding the animacy of the stimulus features.

\section{Bottom-up mechanisms of biological motion}

In the present study, the stimulus derived from the movement of a human hand directed attention in a bottom-up manner toward a potentially forthcoming target location. This finding is consistent with a substantial number of previous studies (e.g., Flanagan \& Johansson, 2003; Shi et al., 2010). The redirection 
of attention when observing the movement of a model or coactor (joint or co-operative action) has been described previously (Welsh et al., 2005). In typical paradigms, the directional movement of a real or virtual human model or the motion of one or two dots representing a human model (Shi et al., 2010; Wang et al., 2014) directs attention toward the relevant side of space, enhancing processing of and response to forthcoming targets. Moreover, belief that both simple and complex displays are derived from animate models can alter movements (arm reaches; Sparks, Douglas, \& Kritikos, 2016; Sparks, Sidari, et al., 2016). Here, however, we argue that biological motion can modify complex attention mechanisms. Using pointing actions, we showed a pattern of RTs to targets consistent with initial shifting of attention in the direction of the cue, then a disengagement from that location - the early enhancement of responses indicating facilitation, and the later slowing consistent with inhibition. Social IOR has been described previously, between participants seated across from each other (e.g., Welsh et al., 2005). Crucially, we showed this IOR effect with a very simple, biological-motion-based stimulus, a single dot. Furthermore, this is the first report of a facilitation effect in the context of social IOR, using a social stimulus.

We can be confident that the salience of the kinematic pattern of the biological motion is what triggers changes in the direction of attention, because when the biological nature of the display was disrupted (inverted or rendered nonbiological), the pattern of facilitation and IOR was abolished. It is likely that the motion of the dot is coded as "biological" by the visuomotor system because it follows movement principles expected for biological organisms. For example, human hand actions tends to follow a somewhat vertically and laterally curved trajectory during reach movements (Viviani \& Flash, 1995). The velocity of the hand slows in the curved parts of the trajectory as the hand travels upward, against gravity, and accelerates in straighter parts of the trajectory. Humans can accurately distinguish the motion of a single dot as "natural" when it moves according to this rule (e.g., Bidet-Ildeil, Orliaguet, Sokolov, \& Pavlova, 2006), probably based on learning through predictive inference and Bayesian coding principles (e.g., Otten, Seth, \& Pinto, 2017).

Conversely, however, inverting the display disrupts the influence of biological motion, even though the velocity profile of the motion in the primary left-right direction was similar. This disruption probably occurs because such inversions impair configural processing of the multiple points moving coherently (Troje \& Westhoff, 2006) and/or disrupt the pattern of motion with respect to gravity (Chang \& Troje, 2008; Thurman \& Lu, 2013). Thus, it is possible that this motion pattern did not activate a representation of human action, nor subsequently trigger reflexive shifts in attention. We can make a similar argument for the nonbiological (transient) motion dot: The lack of (upward) curvature and acceleration of the trajectory likely did not activate a representation of a human action.

\section{Top-down mechanisms that tune the system}

Arguably, the more meaningful set of findings in this present study, however, is that we induced a representation of human action, and thus shifts of attention, through manipulation of animacy belief. That is, top-down factors such as belief of animacy can tune the perception of visual stimuli. The pattern of responses to the targets was consistent with attentional shifts both when the dot motion represented human movement and when its motion was nonbiological but participants believed it represented a human. We speculate that the latter belief effect occurs because social context is relevant to our visuomotor system (Gowen et al., 2016; Longo \& Bertenthal, 2009; Sparks, Sidari, et al., 2016; Stanley et al., 2007). Indeed, Sparks, Sidari, et al. (2016) showed that merely displaying a still image of a human model, without providing an explanation, provides sufficient social context to generate corepresentation and motor imitation effects associated with motor contagion in observers of an adjacent single white dot moving along a vertically curved trajectory. These effects may be due to a preparation of the organism for certain classes of stimuli that occur in our (visual) environment (e.g., Bruner, Postman, \& Rodrigues, 1951) - in the paradigms discussed here, social context. Thus, an image of a human model (Sparks, Sidari, et al., 2016), pro-social words (Sparks et al., 2016), or the animacy belief instructions in the present study prepare the individual for a social context and prime mechanisms leading to social interactions. Otten et al. (2017) postulated that preparation by social contextual factors tunes neural activity, and thus the processing of visual features. The preparation occurs through a proactive hypothesis about the sensory information displayed and prior expectations regarding that information.

In contrast to these postulations regarding belief, Press (2011) pointed out that in action observation paradigms, there is inconclusive evidence that observing the motion of robotic hands while believing them to be human causes changes in the movement of the observer. She speculated that this inconclusive evidence could be a function of the simplicity of the stimulus - decoding of a simple stimuli (such as one or two dots) might use contextual information to solve the problem of decoding and processing. Given greater information, such as a complete PLD, would limit the usefulness of the belief and expectation to the decoding process. We have found previously in an action observation task, however (Kritikos, McTaggart, Painter, \& Bayliss, 2011), that the motion of a hand PLD causes the same extent of ideomotor priming as an image of the same, real hand, which inherently has greater complexity and information than its PLD counterpart. Moreover, biological motion might assist the visuomotor system in precisely those situations in which information is minimal, such as brief displays or attenuated light conditions. In sum, belief regarding the origin of a moving cue could bias 
perception to treat the nonbiological motion stimulus as salient biological motion. Future studies could investigate whether this interacts with the complexity of information available.

Next we address the findings of the computer-generated belief condition. To determine whether instructions alone could engage visuomotor and attentional processes in the nonbiological motion condition, a fifth group of participants responded to cued and uncued targets under nonbiological dot motion conditions. If the dot stimulus in the animacy belief condition generated attentional shifts and engagement of visuomotor processes, rather than the belief of animacy per se, then responses should be faster on uncued than on cued target trials for 600-ms and 1,300-ms SOAs. If, however, the shift of attention was driven by biological motion or belief in the animacy of the dot motion, then the IOR should be abolished, as seen in the nonbiological and inverted biological conditions. Prior to starting the task, participants were instructed that the moving dot was computer generated. The results of this condition provided some evidence that the instructions did prime and alter the processing of the motion stimulusIOR was indeed abolished at the $600-\mathrm{ms}$ SOA, but IOR was evident again at the 1,300-ms SOA. Moreover, RTs were significantly longer to uncued than to cued targets at the 200-ms SOA. Direct comparisons between the animacy belief condition and the computer-generated condition, however, revealed that the influences of these two instructions were distinct. The IOR that was present at the 600 -ms SOA of the animacy belief condition was not present in the computer-generated belief condition. Thus, although instructions regarding the origins of the stimulus could alter and prime the salience of the stimulus, there are relevant differences in the manners in which beliefs of animacy and nonanimacy shape the salience of the stimulus.

Finally, the RTs overall for the computer-generated belief condition were longer than those for the other conditions, including a nonbiological motion condition that consisted of the identical dot motion stimuli, but without the instruction regarding the origin of the stimulus. It is unclear why these longer overall RTs emerged, but we speculate that participants might have been particularly unmotivated during this task, due to the visual display being extremely simplified (a single moving white dot) when it was made clear that the display was computer generated, a fact that would hold little interest or surprise. Reasonably, participants could have been expected to be as unmotivated in the biological motion condition of the main experiment, because they were not told that the dot motion was based on a human finger. Nonetheless, this characteristic of the biological pattern of motion engaged attentional and visuomotor processes and was associated with shorter RTs than in the computer-generated condition. Similarly, participants might have been expected to be equally unmotivated in the nonbiological and inverted-biological motion conditions, but these conditions were presented without explanation or instruction. Thus, it is possible that the computer-generated belief instruction might have suppressed engagement further, leading to slowed RTs. Along with overall slowed responses in this condition, it is possible that the temporal course of facilitation and inhibition or attention altered, such that inhibition manifested only later, at the 1,300-ms SOA, rather than at the $600-\mathrm{ms}$ SOA seen in the biological motion and animacy belief conditions.

\section{The temporal course of attention facilitation and inhibition}

We now turn to the differences in attention facilitation and inhibition, as a function of SOAs, between the present and previous paradigms using biological motion cues. Here we showed facilitation at a $200-\mathrm{ms}$ SOA, but inhibition at 600 ms and 1,300-ms SOAs. In contrast, Shi et al. (2010) and Wang et al. (2014) presented SOAs of only $600 \mathrm{~ms}$ (walker cue onset for $500 \mathrm{~ms}+$ ISI of $100 \mathrm{~ms}$ ) and reported facilitation. We cannot say whether a longer SOA in either of these studies might have resulted in inhibition. As to the facilitation at a 600-ms SOA, however, recall that Shi et al. used a wholebody PLD of a human walking, whereas the cues in Wang et al. were two dots representing two feet. Furthermore, these PLDs remained stationary, in that the collection of moving dots did not actually translate in one or another direction. It is possible that these visual cues of increased complexity require more time to process, leading to delayed facilitation of attention (Lupiáñez, Milliken, Solano, Weaver, \& Tipper, 2001). Conversely, because these cues were more complex, and thus more informative both visually and socially than the single dot used in the present study, they might have engaged attention at the cued location for an extended period (see also Frischen \& Tipper, 2004). Another way of putting this is that the facilitation effects observed in the present study might have been more transient than those in past studies using walking kinematics, leading to a faster disengagement of attention, and so earlier emergence of inhibition.

\section{Conclusions}

In the present study, we showed that a cue (white dot) representing the movement of a human can trigger the engagement and subsequent inhibition of return of attention. Moreover, the same pattern was seen when observers believed the dot represented human movement (animacy belief), even though the motion was nonbiological transient motion. We postulate that social context in the animacy belief condition caused tuning of the processing of the incoming sensory information in a top-down manner, possibly through generating hypotheses about the origin of the information. 
Acknowledgements This project was partially funded by Australian Research Council Discovery Project 130100253 to A.K., and by an NSERC Discovery Grant to T.N.W. We thank Sebastian Rahe for collecting the data in the final condition. The data will be deposited as supplementary documents on ResearchGate.

\section{References}

Alaerts, K., Nackaerts, E., Meyns, P., Swinnen, S. P., \& Wenderoth, N. (2011). Action and emotion recognition from point light displays: An investigation of gender difference. PLoS ONE, 6, e20989. https://doi.org/10.1371/journal.pone.0020989

Atkinson, A. P., Dittrich, W. H., Gemmell, A. J., \& Young, A. W. (2004). Emotion perception from dynamic and static body expressions in point-light and full-light displays. Perception, 33, 717-746. https:// doi.org/10.1068/p5096

Atkinson, M. A., Simpson, A. A., \& Cole, G. G. (2018). Visual attention and action: How cueing, direct mapping, and social interactions drive orienting. Psychological Bulletin \& Review, 25, 1585-1605. https://doi.org/10.3758/s13423-017-1354-0

Atkinson, M. A., Simpson, A., Skarratt, P. A., \& Cole, G. G. (2014). Is social inhibition of return due to action co-representation? Acta Psychologica, 150, 85-93. https://doi.org/10.1016/j.Actpsy.2014. 04.003

Bardi, L., Di Giorgio, E., Lunghi, M., Troje, N., \& Simion, F. (2015). Walking direction triggers visuo-spatial orienting in 6-month-old infants and adults: An eye tracking study. Cognition, 141, 112120. https://doi.org/10.1016/j.cognition.2015.04.014

Bayliss, A. P., di Pellegrino, G., \& Tipper, S. P. (2005). Sex differences in eye gaze and symbolic cueing of attention. Quarterly Journal of Experimental Psychology, 58, 631-650. https://doi.org/10.1080/ 02724980443000124

Bayliss, A. P., Murphy, E., Naughtin, C. K., Kritikos, A., Schilbach, L., \& Becker, S. I. (2013). "Gaze leading": Initiating simulated joint attention influences eye movements and choice behaviour. Journal of Experimental Psychology: General, 142, 76-92. https://doi.org/10. 1037/a0029286

Bidet-Ildeil, C., Orliaguet, J.-P., Sokolov, A. N., \& Pavlova, M. (2006). Perception of elliptic biological motion. Perception, 35, 1137-114. https://doi.org/10.1068/p5482

Blakemore, S.-J., \& Decety, J. (2001). From the perception of action to the understanding of intention. Nature Reviews Neuroscience, 2, 561-567. https://doi.org/10.1038/35086023

Bosbach, S., Prinz, W., \& Kerzel, D. (2004). A Simon effect with stationary moving stimuli. Journal of Experimental Psychology: Human Perception and Performance, 30, 39-55. https://doi.org/10.1037/ 0096-1523.30.1.39

Bruner, J. S., Postman, L., \& Rodrigues, J. (1951). Expectation and the perception of color. American Journal of Psychology, 64, 216-227. https://doi.org/10.2307/1418668

Chang, D. H. F., \& Troje, N. F. (2008). Perception of animacy and direction from local biological motion signals. Journal of Vision, 8(5), 3: 1-10. https://doi.org/10.1167/8.5.3

Clarke, T. J., Bradshaw, M. F., Field, D. T., Hampson, S. E., \& Rose, D. (2005). The perception of emotion from body movement in pointlight displays of interpersonal dialogue. Perception, 34, 1171-1180. https://doi.org/10.1068/p5203

Cutting, J. E., \& Kozlowski, L. T. (1977). Recognizing friends by their walk: Gait perception without familiarity cue. Bulletin of the Psychonomic Society, 9, 353-356. https://doi.org/10.3758/ BF03337021

Doneva, S. P., Atkinson, M. A., Skarratt, P. A., \& Cole, G. G. (2017). Action or attention in social inhibition of return? Psychological Research, 81, 1-12. https://doi.org/10.1016/j.Actpsy.2014.04.003
Flanagan, J. R., \& Johansson, R. S. (2003). Action plans used in action observation. Nature, 424, 769-771. https://doi.org/10.1038/ nature 01861

Frischen, A., \& Tipper, S. P. (2004). Orienting attention via observed gaze shift evokes longer term inhibitory effects: Implications for social interactions, attention, and memory. Journal of Experimental Psychology: General, 133, 516-533. https://doi.org/10.1037/00963445.133.4.516

Gowen, E., Bolton, E., \& Poliakoff, E. (2016). Believe it or not: Moving non-biological stimuli believed to have human origin can be represented as human movement. Cognition, 146, 431-438. https://doi. org/10.1016/j.cognition.2015.10.010

Hommel, B., Chapman, C. S., Cisek, P., Neyedli, H. F., Song, J.-H., \& Welsh T. N. (in press). No one knows what attention is. Attention, Perception, \& Psychophysics.

Johansson, G. (1973). Visual perception of biological motion and a model for its analysis. Perception \& Psychophysics, 14, 195-204. https:// doi.org/10.3758/BF03212378

Kilner, J., de Hamilton, A. F. C., \& Blakemore, S.-J. (2007). Interference effect of observed human movement on action is due to velocity profile of biological motion. Social Neuroscience, 2, 158-166. https://doi.org/10.1080/17470910701428190

Klein, R. M. (2000). Inhibition of return. Trends in Cognitive Sciences, 4, 138-147. https://doi.org/10.1016/S1364-6613(00)01452-2

Kritikos, A., McTaggart, L., Painter, D. R., \& Bayliss, A. P (2011). Something in the way she moves me: Morphology and motion of observed goal-directed and pantomimed actions. Attention, Perception, \& Psychophysics, 74, 36-42. https://doi.org/10.3758/ s13414-011-0228-1

Longo, M. R., \& Bertenthal, B. I. (2009). Attention modulates the specificity of automatic imitation to human actors. Experimental Brain Research, 192, 739-744. https://doi.org/10.1007/s00221-008-16491655

Lupiáñez, J., Milliken, B., Solano, C., Weaver, B., \& Tipper, S. P. (2001). On the strategic modulation of the time course of facilitation and inhibition of return. Quarterly Journal of Experimental Psychology, 54A, 753-773. https://doi.org/10.1080/02724980042000453

Meegan, D. V., \& Tipper, S. P. (1998). Reaching into cluttered visual environments: Spatial and temporal influences of distracting objects. Quarterly Journal of Experimental Psychology, 51A, 225-249.

Neyedli, H. F., \& Welsh, T. N. (2012). The process of facilitation and inhibition in a cue-target paradigm: Insight from movement trajectory deviations. Acta Psychologica, 139, 159-165. https://doi.org/ 10.1016/j.Actpsy.2011.11.001

Otten, M., Seth, A. K., \& Pinto, Y. (2017). A social Bayesian brain: How social knowledge can shape visual perception. Brain and Cognition, 112, 69-77. https://doi.org/10.1016/j.bandc.2016.05.002

Posner, M. I., \& Cohen, Y. (1984). Components of visual orienting. In H. Bouma \& G. G. Bouwhuis (Eds.), Attention and performance X: Control of language processes (pp. 531-554). Hillsdale, NJ: Erlbaum.

Press, C. (2011). Action observation and robotic agents: Learning and anthropomorphism. Neuroscience \& Biobehavioral Reviews, 35, 1410-1418. https://doi.org/10.1016/j.neubiorev.2011.03.004

Shi, J., Weng, X., He, S., \& Jiang, Y. (2010). Biological motion cues trigger reflexive attentional orienting. Cognition, 117, 348-354. https://doi.org/10.1016/j.cognition.2010.09.001

Song, J.-H., \& Nakayama, K. (2009). Hidden cognitive states revealed in choice reaching tasks. Trends in Cognitive Sciences, 13, 360-366. https://doi.org/10.1016/j.tics.2009.04.009

Sparks, S., Douglas, T., \& Kritikos, A. (2016). Verbal social primes alter motor contagion during action observation. Quarterly Journal of Experimental Psychology, 69, 1041-1048. https://doi.org/10.1080/ 17470218.2015.1113304

Sparks, S., Sidari, M., Lyons, M., \& Kritikos, A. (2016). Pictures of you: Dot stimuli cause motor contagion in presence of a still human form. 
Consciousness and Cognition, 45, 135-145. https://doi.org/10. 1016/j.concog.2016.08.004

Stanley, J., Gowen, E., \& Miall, R. C. (2007). Effects of agency on movement interference during observation of a moving dot stimulus. Journal of Experimental Psychology: Human Perception and Performance, 33, 915-926. https://doi.org/10.1037/0096-1523.33. 4.915

Thurman, S. M., \& Lu, H. (2013). Complex interactions between spatial, orientation, and motion cues for biological motion perception across visual space. Journal of Vision, 13(2), 8. https://doi.org/10.1167/13. 2.8

Tipper, S. P., Lortie, C., \& Baylis, G. C. (1992). Selective reaching: Evidence for action-centred attention. Journal of Experimental Psychology: Human Perception and Performance, 18, 891-905. https://doi.org/10.1037/0096-1523.18.4.891

Tipper, S. P., Weaver, B., Jerreat, L. M., \& Burak, A. L. (1994). Objectbased and environment-based inhibition of return of visual attention. Journal of Experimental Psychology: Human Perception and Performance, 20, 478-499. https://doi.org/10.1037/0096-1523.20. 3.478

Troje, N. F. (2002) Decomposing biological motion: A framework for analysis and synthesis of human gait patterns. Journal of Vision, 2(5), 2:371-387. https://doi.org/10.1167/2.5.2

Troje, N. F., \& Westhoff, C. (2006). The inversion effect in biological motion perception: Evidence for a "life detector"? Current Biology, 16, 821-824. https://doi.org/10.1016/j.cub.2006.03.022

Ulloa, J. L., Marchetti, C., Taffou, M., \& George, N. (2015). Only your yes tell me what you like: Exploring the liking effect induced by other's gaze. Cognition and Emotion, 29, 460-470. https://doi.org/ 10.1080/02699931.2014.919899

Viviani, P., \& Flash, T. (1995). Minimum-jerk, two-thirds power law, and isochrony: Converging approaches to movement planning. Journal of Experimental Psychology: Human Perception and Performance, 21, 32-53. https://doi.org/10.1037/0096-1523.21.1.32

Wang, L., Yang, X., Shi, J., \& Jiang, Y. (2014). The feet have it: Local biological motion cues trigger reflexive attentional orienting in the brain. NeuroImage, 84, 217-224. https://doi.org/10.1016/j. neuroimage.2013.08.041

Welsh, T. N., Chandler-Mather, N., Sparks, S., \& Kritikos, A. (2019). Title of paper. Manuscript in preparation.

Welsh, T. N., \& Elliott, D. (2004). Movement trajectories in the presence of a distracting stimulus: Evidence for a response activation model of selective reaching. Quarterly Journal of Experimental Psychology, 57, 1031-1057. https://doi.org/10.1080/ 02724980343000666

Welsh, T. N., Elliott, D., Anson, J. G., Dhillon, V., Weeks, D. J., Lyons, J. L., \& Chua, R. (2005). Does Joe influence Fred's action? Inhibition of return across different nervous systems. Neuroscience Letters, 385, 99-104. https://doi.org/10.1016/j.neulet.2005.05.013

Welsh, T. N., Elliott, D., \& Weeks, D. J. (1999). Hand deviations toward distractors: Evidence for response competition. Experimental Brain Research, 127, 207-212. https://doi.org/10.1007/s002210050

Welsh, T. N., Higgins, L., Ray, M., \& Weeks, D. J. (2007). Seeing versus believing: Is believing sufficient to activate the processes of response co-representation? Human Movement Science, 26, 853866. https://doi.org/10.1016/j.humov.2007.06.003

Welsh, T. N., Lyons, J., Weeks, D. J., Anson, J. G., Chua, R., Mendoza, J., \& Elliott, D. (2007). Within-and between-nervous-system inhibition of return: Observation is as good as performance. Psychonomic Bulletin \& Review, 14, 950-956. https://doi.org/10.3758/ BF03194127

Welsh, T. N., McDougall, L. M., \& Weeks, D. J. (2009). The performance and observation of action shape future behaviour. Brain and Cognition, 71, 64-71. https://doi.org/10.1016/j.bandc.2009.04.001

Welsh, T. N., \& Weeks, D. J. (2010). Visual selective attention and action. In D. Elliott \& M. A. Khan (Eds.), Vision and goal-directed movement: Neurobehavioral perspectives (pp. 39-58). Champaign, IL: Human Kinetics.

Publisher's note Springer Nature remains neutral with regard to jurisdictional claims in published maps and institutional affiliations. 